\title{
ON A CLASS OF FUNCTIONS OF COMPLEX ORDER
}

\author{
S. ABDUL HALIM
}

\begin{abstract}
Denote by $\mathcal{R}(b)$, the class of normalized analytic functions $f$ which satisfies $\operatorname{Re}(1+$ $\left.\frac{1}{b}\left(f^{\prime}(z)-1\right)\right)>0$, for $z \in D=\{z:|z|<1\}$ and $b$ a non-zero complex number. In this paper, some results concerning functions belonging to this class are being considered.
\end{abstract}

\section{Introduction}

Denote by $A$ the class of functions $f$ which are normalized such that $f(0)=f^{\prime}(0)-1=$ 0 and analytic in the unit $\operatorname{disc} D=\{z:|z|<1\}$. Also denote by $S$, the subclass of $A$ consisting of all univalent functions in $D$.

Let $P$ be the class of functions $p(z)=1+\sum_{n=1}^{\infty} c_{n} z^{n}$ which are analytic and also have a positive real part in $D$. A function $f$ is said to be starlike if and only if, for $z \in D$, $\operatorname{Re} \frac{z f^{\prime}(z)}{f(z)}>0$. The well-known class of starlike functions which we shall denote by $S^{*}$ is a subclass of $S$ and have been extensively studied, see [3], [4] and [14]. In [13], Nasr and Aouf intorduced the class consisting of functions which are starlike of complex order. Functions $f$ belonging to this class, say $S^{*}(b)$ are those which satisfied the following condition:

$$
\operatorname{Re}\left(1+\frac{1}{b}\left(\frac{z f^{\prime}(z)}{f(z)}-1\right)\right)>0, \quad z \in D \text { and } b \neq 0, b \in \mathbb{C}
$$

J. W. Alexander in [1] intorduced the class $\mathcal{R}$ consisting of normalized analytic functions whose derivative has a positive real part, and proved that for $z \in D$, if $f$ belongs to $\mathcal{R}$ then $f$ is univalent, i.e. $\mathcal{R} \subset S$. This class $\mathcal{R}$ has also been considered quite extensively by Macgregor (see [8]). We now introduce an extension of this class via the following definition.

Definition 1.1. A function $f \in A$ is said to belong to the class $\mathcal{R}(b)$, if and only if, for $z \in D$

$$
\operatorname{Re}\left(1+\frac{1}{b}\left(f^{\prime}(z)-1\right)\right)>0
$$

where $b$ is a non-zero complex number.

Received September 28, 1998; revised December 28, 1998.

1991 Mathematics Subject Classification. Primary 30C45.

Key words and phrases. Univalent functions, starlike of complex order, functions of complex order, integral operator. 
Note that $\mathcal{R}(1) \equiv \mathcal{R}$.

In this paper, the author gives some results concerning functions in $\mathcal{R}(b)$.

\section{Preliminary Results}

Lemma 2.1 [6]. For any complex number $\mu$ and $p \in P$ given by $p(z)=1+c_{1} z+$ $c_{2} z^{2}+\cdots$,

$$
\left|c_{2}-\mu c_{1}^{2}\right| \leq 2 \max \{1,|1-2 \mu|\} .
$$

Lemma $2.2[9]$. Suppose that the function $\psi: \mathbb{C}^{2} \times D \rightarrow \mathbb{C}$ satisfies the condition

$$
\operatorname{Re} \psi(i x, y, z) \leq 0
$$

for all $x, y \leq-\left(1+x^{2}\right) / 2$ and all $z \in D$.

If the function $p(z)$ is analytic in $D$, with $p(0)=1$ and if

$$
\operatorname{Re} \psi\left(p(z), z p^{\prime}(z), z\right)>0 \quad \text { for } z \in D
$$

then $\operatorname{Re} p(z)>0$ in $D$.

\section{Some Properties of $\mathcal{R}$}

Theorem 3.1. Let $f \in \mathcal{R}(b)$. If Re $b \geq|b|^{2}$ then $f$ is univalent.

Proof. First, we write $b=|b| e^{i \sigma}$. Now, since $\operatorname{Re} b \geq|b|^{2}$, it follows that $|b| \leq 1$ and $|\sigma|<\frac{\pi}{2}$. Next, inequality (1) implies that

$$
\operatorname{Re} \frac{f^{\prime}(z)}{b}>\operatorname{Re}\left(\frac{1}{b}\right)-1 \geq 0,
$$

provided $\operatorname{Re} b \geq|b|^{2}$.

Thus, we have

$$
\operatorname{Re} e^{-i \sigma} f^{\prime}(z)>0
$$

and hence $f$ is univalent.

Remark 3.1. The question of whether the converse of Theorem 1 is true or not remains open. On the other hand, one can construct a non-univalent function belonging to $\mathcal{R}(b)$ for which $b$ is outside this disc.

Our next theorem looks at distortion results for $f \in \mathcal{R}(b)$.

Theorem 3.2. Let $f \in \mathcal{R}(b)$. Then for $z=r e^{i \theta} \in D$,

(i) $2|b|(\log (1+r)-r)+r \frac{\mathrm{Re} b}{|b|} \leq|f(z)| \leq(1-2|b|) r-2|b| \log (1-r)$, 
(ii) $\frac{\operatorname{Re} b}{|b|}-\frac{2 r|b|}{1+r} \leq\left|f^{\prime}(z)\right| \leq(1-2|b|)+\frac{2|b|}{1-r}$.

Proof. From (1), we write

$$
f^{\prime}(z)=b(p(z)-1)+1
$$

where $p(z)=1+\sum_{n=1}^{\infty} c_{n} z^{n} \in P$. Thus for $z=r e^{i \theta} \in D$,

$$
\begin{aligned}
|p(z)|-1 & \leq \sum_{n=1}^{\infty}\left|c_{n} z^{n}\right| \\
& \leq 2 \sum_{n=1}^{\infty} r^{n} \\
& =-1+\frac{1+r}{1-r} .
\end{aligned}
$$

Here, we use the known result that $\left|c_{n}\right| \leq 2$, (2) and (3) then gives

$$
\begin{aligned}
|f(z)| & =\left|z+b \int_{0}^{z}(p(t)-1) d t\right| \\
& \leq r-2|b|(r+\log (1-r)),
\end{aligned}
$$

which is the upper bound in (i).

To prove the lower bound in (i), write

$$
\begin{aligned}
\left|\frac{f(z)}{z}\right| & =\left|\frac{1}{z} \int_{0}^{z} f^{\prime}(t) d t\right| \\
& =|b|\left|\frac{1}{z} \int_{0}^{z}\left(p(t)-1+\frac{1}{b}\right) d t\right| \\
& \geq|b| \operatorname{Re}\left\{\frac{1}{z} \int_{0}^{z}\left(p(t)-1+\frac{1}{b}\right) d t\right\} .
\end{aligned}
$$

Next, write $t=\rho e^{i \theta}$, we have

$$
\begin{aligned}
|f(z)| & \geq r|b|\left\{\frac{1}{r} \int_{0}^{r} \operatorname{Re}\left(p\left(\rho e^{i \theta}\right)-1+\frac{1}{b}\right) d \rho\right\} \\
& \geq|b|\left\{\int_{0}^{r}\left(\frac{1-\rho}{1+\rho}\right) d \rho-r+r \operatorname{Re} \frac{1}{b}\right\} \\
& =-2 r|b|+2|b| \log (1+r)+\frac{r}{|b|} \operatorname{Re} b .
\end{aligned}
$$

The upper bound in (ii) follows at once from (2) and (3).

Finally, for the lower bound of (ii), write

$$
\left|f^{\prime}(z)\right|=|b|\left|p(z)-1+\frac{1}{b}\right|
$$


which gives the result.

$$
\begin{aligned}
& \geq|b| \operatorname{Re}\left(p(z)-1+\frac{1}{b}\right) \\
& \geq|b|\left(\frac{1-r}{1+r}-1+\frac{\operatorname{Re} b}{|b|^{2}}\right)
\end{aligned}
$$

Thoerem 3.3. Let $f \in \mathcal{R}(b)$. Then for $z=r e^{i \theta} \in D$,

(i) $\operatorname{Re} f^{\prime}(z) \geq 1-\frac{2 r}{1-r^{2}}(|b|-r \operatorname{Re} b)$,

(ii) $\operatorname{Re} \frac{f(z)}{z} \geq 1-2 \operatorname{Re} b+\frac{\log (1=r}{r}(|b|+\operatorname{Re} b)+\frac{\log (1-r)}{r}(|b|+\operatorname{Re} b)$.

We note that for $b=1$, (ii) reduces to a result proved by Hallenbeck [5]:

Proof. in [12], Nasr and Aouf showed that if $p \in P$, then

$$
\operatorname{Re}[b(p(z)-1)+1] \geq 1-\frac{2 r}{1-r^{2}}(|b|-\operatorname{Re} b) .
$$

hence, using (2), (i) follow trivially.

Next,

Now write $t=\rho e^{i \theta}$, we have

$$
\operatorname{Re} \frac{f(z)}{z}=\operatorname{Re}\left\{\frac{1}{z} \int_{0}^{z}[b p(t)+1-b] d t\right\} .
$$

$$
\begin{aligned}
\operatorname{Re} \frac{f(z)}{z} & \geq \frac{1}{r} \int_{0}^{z}\left(1-\frac{2|b| \rho}{1-\rho^{2}}+\frac{2 \operatorname{Re} b \rho^{2}}{1-\rho^{2}}\right) d \rho \\
& =1+\frac{|b|}{r} \log \left(1-r^{2}\right)+2 \frac{\operatorname{Re} b}{r}\left(\frac{\log (1+r)}{2}-\frac{\log (1-r)}{2}-r\right)
\end{aligned}
$$

which on simplication completes the proof.

\section{Coefficient Results}

We next consider coefficient estimates.

Theorem 4.1. Let $f \in \mathcal{R}(b)$ with $f(z)=z+\sum_{n=2}^{\infty} a_{n} z^{n}$. Then

(i) $\left|a_{n}\right| \leq \frac{2|b|}{n}$, for $n \geq 2$,

and (ii) $\left|a_{3}-\mu a_{2}^{2}\right| \leq \frac{2|b|}{3} \max \left\{1,\left|1-\frac{3}{2} \mu b\right|\right\}$,

where $\mu$ is any complex number. The inequalities are sharp.

Proof. Since $f \in \mathcal{R}(b)$, there exists $p \in P$ such that for $p(z)=1+c_{1} z+c_{2} z^{2}+\cdots$ (2) holds. Thus

$$
1+\sum_{n=2}^{\infty} n a_{n} z^{n-1}=1+\sum_{n=1}^{\infty} b c_{n} z^{n} .
$$


Equating the coefficients in (6) gives

$$
n a_{n}=b c_{n-1}
$$

and so (i) follows immediately, since $\left|c_{n-1}\right| \leq 2$ for $n \geq 2$. Finally using Lemma 2.1, it follows that

$$
\begin{aligned}
\left|a_{3}-\mu a_{2}^{2}\right| & =\frac{|b|}{3}\left|c_{2}-\frac{3 \mu}{4} b c_{1}^{2}\right| \\
& \leq \frac{2|b|}{3} \max \left\{1,\left|1-\frac{3}{2} \mu b\right|\right\} .
\end{aligned}
$$

(4) and (5) are sharp for $f_{1}(z)=z-2 b(z+\log (1-z))$ in the case $|2-3 \mu b|>2$. For $|2-3 \mu b| \leq 2$, inequality (5) is sharp for $f_{2}$ given by

$$
f_{2}^{\prime}(z)=b\left\{\frac{1+z^{2}}{1-z^{2}}-1\right\}+1 .
$$

\section{Integral Operators}

In this final section, we look at integral operator which preserves the class $\mathcal{R}(b)$.

Definition 5.1. Suppose that $g \in A$ with $g(z) \neq 0$ for $z \in D$. for $z \in D$, define the integral operator

$$
F(z)=\frac{1+c}{g(z)^{c}} \int_{0}^{z} f(t)[g(t)]^{c-1} g^{\prime}(t) d t
$$

where $c>-1$.

Putting $g(z) \equiv z$ and $c=1$ in (7) gives the operator first introduced by Libera in [7]. This was then followed by Bernardi [2] who generalised Libera's operator by introducing the constant $c$ for $c \in \mathbb{N}$. Since then, many people have extended the results obtained for Libera and Bernardi integral operators. See for example [15] and [16]. Some authors considered other similar forms of integral operators such as that given in (7) for various subclasses of $S$. This include Mocanu [11], Miller et. al [10] and Selinger [17].

We now proceed to state our result.

Theorem 5.1. Let $g \in S^{*}$ with $Q(z)=\frac{g(z)}{z g^{\prime}(z)}$, for $z \in D$. If $f \in \mathcal{R}(b)$ and

$$
\begin{aligned}
& \operatorname{Re} Q(z) \cdot \operatorname{Re}\left[Q(z)+\frac{2}{b}(b-1)\left(z Q^{\prime}(z)+Q(z)-1\right)\right] \\
\geq & \left\{\operatorname{Im}\left(z Q^{\prime}(z)+Q(z)+c\right\}^{2},\right.
\end{aligned}
$$

for $z \in D$, then the integral operator $F$ given by (7) also belongs to $\mathcal{R}(b)$.

We note that, in the case $b=1$, this result reduces to the one given by Selinger [17]. To prove this theorem, we require Lemma 2.2 . 
Proof. For $F$ given by (7), it can easily be deduced that

$$
z Q(z) F^{\prime}(z)+c F(z)=(1+c) f(z) .
$$

Differentiating (9) again, gives

$$
z Q(z) F^{\prime \prime}(z)+F^{\prime}(z)\left[z Q^{\prime}(z)+Q(z)+c\right]=(1+c) f^{\prime}(z) .
$$

Now, since $f \in \mathcal{R}(b)$ and $c>-1$, thus

$$
\operatorname{Re}\left\{z Q(z) h^{\prime}(z)+\left(z Q^{\prime}(z)+Q(z)+c\right) h(z)+\frac{1}{b}(1-b)\left(z Q^{\prime}(z)+Q(z)-1\right)\right\}>0,
$$

where $h(z)=1+\frac{1}{b}\left(F^{\prime}(z)-1\right)$.

For convenience, we introduce functions $B$ and $D$, and rewrite (10) as follows: $\operatorname{Re}\left\{z Q(z) h^{\prime}(z)+B(z) h(z)+D(z)\right\}>0$.

We next show that for $g \in S^{*}$ with inequality (8) true, $h \in P$ and this in turn implies that $F \in \mathcal{R}(b)$. We do this by using Lemma 2.2. First, define

$$
\psi(i x, y, z)=y Q(z)+i x B(z)+D(z) .
$$

In order to use Lemma 2.2, we need to verify that $\forall x \in \mathbb{R}, y \leq \frac{-\left(1+x^{2}\right)}{2}$ and $z \in D$, $\operatorname{Re} \psi(i x, y, z) \leq 0$.

$$
\begin{aligned}
\operatorname{Re} \psi(i x, y, z) & =y \operatorname{Re} Q(z)-x \operatorname{Im} B(z)+\operatorname{Re} D(z) \\
& \leq \frac{-\left(1+z^{2}\right)}{z} \operatorname{Re} Q(z)-x \operatorname{Im} B(z)+\operatorname{Re} D(z) ;
\end{aligned}
$$

for $y \leq \frac{-\left(1+x^{2}\right)}{2}$ and $g \in S^{*}$.

Therefore,

$$
\operatorname{Re} \psi(i x, y, z) \leq-x^{2} \frac{\operatorname{Re} Q(z)}{2}-x \operatorname{Im} B(z)+\operatorname{Re}\left(D(z)-\frac{Q(z)}{z}\right) .
$$

Since, inequality (8) is true, i.e. $\operatorname{Re} Q(z) \cdot \operatorname{Re}[Q(z)-2 D(z)] \geq[\operatorname{Im} B(z)]^{2}$, thus we conclude that $\operatorname{Re} \psi(i x, y, z) \leq 0$. Furthermore, since (10) implies that $\operatorname{Re}\left(h(z), z h^{\prime}(z), z\right)>0$, for $z \in D$, thus by Lemma $2.2, h \in P$ and this completes our proof.

\section{Acknowledgement}

The author is very much grateful and thankful to the referee for his comments and suggestions which resulted in an improvement of the paper. 


\section{References}

[1] J. W. Alexander, Functions which map the interior of the unit circle upon simple regions, Ann. of Math. 17(1915), 12-22.

[2] S. D. Bernardi, Convex and starlike univalent functions, Trans. Amer. Math. Soc. 1.35(1969), 429-446.

[3] P. L. Duren, Univalent Functions, Springer Verlag, Berlin, 1983.

[4] A. W. Goodman, Univalent Functions, Vol. I, II, Polygonal Publishing House, Washington, 1983.

[5] D. J. Hallenbeck, Convex hulls and extreme points of some families of univalent functios, Trans. Amer. Math. Soc. 192(1974), 285-292.

[6] F. R. Keogh and E. P. Merkes, A coefficient inequality for certain classes of analytic functions, Proc. Amer. Math. Soc. 20(1969), 8-12.

[7] R. J. Libera, some classes of regular univalent functions, Proc. Amer. Maths. Soc. 16(1965), 755-758.

[8] T. H. Macgregor, Functions whose derivative has a positive real part, Trans. Amer. Math. Soc. 9(1962), 532-537.

[9] S. S. Miller and P. T. Mocanu, Second order differential inequalities in the complex plane, J. Math. Anal. Appl. 65(1978), 289-305.

[10] S. S. Miller, P. T. Mocanu and M. O. Reade, Starlike integral operators, Pacific J. Math. $79(1978), 157-168$.

[11] P. T. Mocanu, On a close-to-convexity preserving integral operator, Studia Univ. BabesBolyai, Math. 32(1987), 53-56.

[12] M. A. Nasr and M. K. Aouf, Radius of convexity for the class of starlike functions of complex order, Bull. Fac. Sci. Assiut Univ. 12(1983), 153-159.

[13] - Starlike functions of complex order, J. of Natural Sciences and Mathematics 25 (1985), $1-12$.

[14] R. Nevalinna, Über die konforme Abbilung von Sterngebieten, Översikt av Finska-Vetenskap Soc. Förh. 63(1921), 1-21.

[15] H. M. Srivastava and S. Owa (Editors), Current Topics in Analytic Function Theory, World Scientific Publishing Company, Singapore, new jersey, london and Hong Kong, 1992.

[16] Rosihan M. Ali and D. K. Thomas, Proc. Japan Acad. 67A (1991), 319-321.

[17] V. Selinger, Some integral operators preserving certain geometric properties, Rev. Roumaine Math-Pures Appl.'33(1988), 10, 889-900.

Institute of Mathematical Sciences, University of Malaya, 50603 Kuala Lumpur, Malaysia. 